\title{
MIXED HODGE STRUCTURES ON HOMOTOPY GROUPS
}

\author{
BY RICHARD M. HAIN ${ }^{1}$
}

In [3] Deligne defined mixed Hodge structures (M.H.S.'s) and showed that the cohomology of every algebraic variety over $\mathbf{C}$ has a natural M.H.S. Morgan [12], using Sullivan's minimal models, showed that the rational homotopy Lie algebra and rational homotopy type of every smooth variety have natural M.H.S.'s. In this note we announce an extension of mixed Hodge theory to arbitrary varieties and homotopy fibers of morphisms between varieties. The latter is a major step in extending asymptotic Hodge theory to homotopy groups and periods of iterated integrals. The bar construction and KuoTsai Chen's iterated integrals [1] provide the link between Hodge theory and homotopy groups. Some of the results announced have been distributed in preprint form [7]. Proofs of the results stated will be published elsewhere.

Because the higher homotopy groups of a non-nilpotent topological space are inaccessible to rational homotopy theory, we make the following definition. The homotopy Lie algebra of a pointed topological space $(X, x)$ is the graded Lie algebra $\mathfrak{g}_{\text {. }}(X, x)$ where $\mathfrak{g}_{0}(X, x)$ is the Malcev Lie algebra associated with $\pi_{1}(X, x)$ and, when $k \geq 1$,

$$
\mathfrak{g}_{k}(X, x)= \begin{cases}\pi_{k+1}(X, x) \otimes \mathbf{Q} & \text { if }(X, x) \text { is nilpotent } \\ 0 & \text { otherwise }\end{cases}
$$

The class of nilpotent spaces includes simply connected spaces and topological groups. There is a Hurewicz homomorphism

$$
\mathfrak{g}_{k}(X, x) \rightarrow H_{k+1}(V, \mathbf{Q}) .
$$

THEOREM 1. If $(V, x)$ is a pointed algebraic variety, then the homotopy Lie algebra of $(V, x)$ has a M.H.S. that is functorial with respect to morphisms of pointed varieties and such that

(a) the bracket is a morphism of M.H.S.'s.

(b) The Hurewicz homomorphism is a morphism of M.H.S.'s. Moreover, if $(V, W, x)$ is a pair of simply connected varieties, then $\pi .(V, W, x)$ has a natural M.H.S. and the long exact sequence of the pair is a long exact sequence of M.H.S.'s.

If $(V, x)$ is simply connected, then the M.H.S. on $\pi_{k}(V, x)$ does not depend on the basepoint $x$. However, if $V$ is not simply connected, this is not the case.

Received by the editors April 25, 1985.

1980 Mathematics Subject Classification (1985 Revision). Primary 14F35, 14F40; Secondary 55P62.

${ }^{1}$ Supported in part by grants MCS-8201642 and DMS-8401175 from the National Science Foundation. 
Denote the integral group ring of a group $G$ by $\mathbf{Z} G$. The augmentation ideal $J$ is the kernel of the augmentation $\mathbf{Z} G \rightarrow \mathbf{Z}: g \rightarrow 1$. One can also show that if $(V, x)$ is a pointed variety, then for each $s$, the truncated group ring

$$
\mathbf{Z} \pi_{1}(V, x) / J^{s}
$$

has a M.H.S.

The following theorem is an improvement of a result of the author by M. Pulte [13].

THEOREM 2. Suppose that $X$ and $Y$ are smooth projective curves and that $x \in X$ and $y \in Y$. Suppose that

$$
\Phi: \mathbf{Z} \pi_{1}(X, x) / J^{3} \rightarrow \mathbf{Z} \pi_{1}(Y, y) / J^{3}
$$

is a ring isomorphism that commutes with the augmentations. If $\Phi$ induces an isomorphism of M.H.S.'s, then for all but at most two exceptional points $x$ of $X$, there exists a biholomorphism $\phi: X \rightarrow Y$ such that $\phi(x)=y$.

Presumably these exceptional points do not exist. The previous result is related to the study of certain algebraic 1-cycles in $\operatorname{Jac}(X)$ via the work of Bruno Harris [11]. The details of this connection will appear in [13].

It is sometimes convenient to have a M.H.S. on the rational homotopy type of a variety.

THEOREM 3 ( $C f$. [12] when $V$ is smooth). If $(V, x)$ is a pointed variety, then the rational Lie algebra model and rational minimal model of $(V, x)$ have (not necessarily unique) M.H.S.'s.

Using this result, one can find nontrivial restrictions on the rational homotopy types of projective varieties.

THEOREM 4. There exists a simply connected finite $C W$-complex $X$ whose integral cohomology ring is isomorphic to that of a simply connected projective variety, but $X$ does not have the rational homotopy type of any projective variety.

THEOREM 5. If $X$ is a complete (e.g. projective) variety whose rational cohomology ring satisfies Poincaré duality, then $X$ is formal.

There are many such varieties that are not smooth. The main result of [4] follows from Theorem 5. The following interesting corollary of Theorem 5 and a result from [9] was pointed out to me by S. Halperin.

COROLlARY 6. Suppose that $V$ is a simply connected complete variety. If $\pi_{k}(V)$ is torsion when $k$ is sufficiently large, then $V$ is formal.

In the homotopy category, every continuous map $f: X \rightarrow Y$ can be canonically replaced by a fibration $\pi: E_{f} \rightarrow Y$ where $E_{f}$ is homotopy equivalent to $X$. The fiber $\pi^{-1}(y)$ is called the homotopy fiber of $f$ over $y$ and will be denoted by $E_{f}(y)$. If $Y$ is path connected, then all homotopy fibers are homotopy equivalent. For example, if $Y$ is an Eilenberg-Mac Lane space $K(\pi, 1)$, then the homotopy fiber of $f$ is the total space of the covering of $X$ determined by the kernel of $\pi_{1}(X) \rightarrow \pi_{1}(Y)$.

Set $X_{y}=f^{-1}(y)$. There is a natural inclusion $X_{y} \rightarrow E_{f}(y)$, which is a weak equivalence if $f$ is a fibration. 
THEOREM 7. Suppose that $f: X \rightarrow Y$ is a morphism of algebraic varieties. If $E_{f}(y)$ is path connected and $\pi_{1}(Y, y)$ acts unipotently on $H^{\cdot}\left(E_{f}(y) ; \mathbf{Q}\right)$, then the cohomology and the rational homotopy Lie algebra (and types) of $E_{f}(y)$ have natural M.H.S.'s. Furthermore, the restriction map

$$
H^{\bullet}\left(E_{f}(y)\right) \rightarrow H^{\bullet}\left(X_{y}\right)
$$

and the monodromy representation

$$
H^{\bullet}\left(E_{f}(y)\right) \otimes \mathfrak{g}_{0}(Y, y) \rightarrow H^{\bullet}\left(E_{f}(y)\right)
$$

are morphisms of M.H.S.'s. If $X$ and $Y$ are simply connected, then the long exact sequence of homotopy groups associated with the fibration $E_{f}(y) \rightarrow X \rightarrow$ $Y$ is a sequence of M.H.S.'s.

This theorem is true for spaces more general than algebraic varieties. Loosely speaking, $X$ and $Y$ may be replaced by any topological spaces whose cohomology and homotopy have natural M.H.S.'s. In [5] it is shown that the cohomology and homotopy of a deleted tubular neighbourhood of a subvariety of a smooth variety have natural M.H.S.'s.

If $f: X \rightarrow \Delta$ is a local degeneration of projective varieties, then after a base change if necessary, we may assume that the monodromy representation is unipotent. Theorem 7, combined with Steenbrink's work [14], then yields the next result.

THEOREM 8. If $f: X \rightarrow \Delta$ is a degeneration of projective varieties with unipotent monodromy, then the M.H.S. on the homotopy fiber of $X^{*} \rightarrow \Delta^{*}$ is the limit M.H.S. on the cohomology of the generic fiber of $f$. Moreover, if $\sigma: \Delta \rightarrow X$ is a section, then there is a limit M.H.S. on the homotopy Lie algebra of $\left(X_{t}, \sigma(t)\right)$ when $t \neq 0$ such that the natural map

$$
\mathfrak{g} \cdot\left(X_{0}, \sigma(0)\right) \rightarrow \mathfrak{g} \cdot\left(X_{t}, \sigma(t)\right)_{\lim }
$$

is a morphism of M.H.S.'s.

One can show that the Hodge filtration on the homotopy Lie algebra varies holomorphically with $(X, x)$ and satisfies Griffiths transversality. Also, in joint work with Zucker [8], we show that the variation of M.H.S. over a variety $V$ whose fiber over $x$ is $\mathfrak{g}_{0}(V, x)$ classifies all variations of M.H.S. over $V$ with unipotent monodromy, a result conjectured by Deligne.

9. Sketch of proofs. The first step is to show that if $A^{\bullet}$ is a mixed Hodge complex (M.H.C.) that is also a d.g.a. (a multiplicative M.H.C.) and if $M^{\bullet}$, $N^{\bullet}$ are M.H.C.'s that are right and left $A^{\bullet}$ modules respectively, then Chen's reduced bar construction $\bar{B}\left(M^{\bullet}, A^{*}, N^{*}\right)[2]$ is also a M.H.C. For Theorem 1, we need to show that if $A^{*}$ is a multiplicative M.H.C. that is augmented, then the usual bar construction $B\left(A^{\circ}\right)$ and its indecomposables $Q B\left(A^{\circ}\right)$ are also M.H.C.'s.

The second step is to construct for each algebraic variety $V$ a commutative multiplicative M.H.C. whose rational part has the same homotopy type as the Thom-Sullivan rational de Rham complex of $V$. This is done by taking $A^{*}$ to be a collection of compatible forms on the geometric realization of a hypercovering of $V$. 
Theorems 1 and 7 now follow from results in $[\mathbf{1}, \mathbf{6}, \mathbf{1 0}]$ and standard results from algebraic topology.

ACKNOWLEDGEMENTS. The author would like to thank Jim Carlson, Alan Durfee, Steve Halperin and Steve Zucker for helpful conversations.

\section{REFERENCES}

1. K.-T. Chen, Iterated path integrals, Bull. Amer. Math. Soc. 83 (1977), 831-879.

2. __ Reduced bar constructions on de Rham complexes, Algebra, Topology, and Category Theory, Academic Press, New York, 1976.

3. P. Deligne, Théorie d'Hodge. II, Inst. Hautes Études Sci. Publ. Math. 40 (1971), 5-58 and III, 44 (1975), 5-77.

4. P. Deligne, P. Griffiths, J. Morgan and D. Sullivan, Real homotopy theory of Kähler manifolds, Invent. Math. 29 (1975), 245-274.

5. A. Durfee and R. Hain, Mixed Hodge structures on the homotopy of links of singular points, preprint, April, 1985.

6. R. Hain, Iterated integrals and homotopy periods, Mem. Amer. Math. Soc. no. 291 (1984).

7. The de Rham homotopy theory of complex algebraic varieties, preprint, January 1984.

8. R. Hain and S. Zucker, Unipotent variations of mixed Hodge structures (preprint, November 1985).

9. S. Halperin, Finiteness in the minimal model of Sullivan, Trans. Amer. Math. Soc. 230 (1977), 173-199.

10. __ Lectures on minimal models, Mem. Soc. Math. France no. 9/10 (1983), 261 pp.

11. B. Harris, Harmonic volumes, Acta Math. 150 (1983), 91-123.

12. J. Morgan, The algebraic topology of smooth algebraic varieties, Inst. Hautes Études Sci. Publ. Math. 48 (1978), 137-204.

13. M. Pulte, Thesis, University of Utah, 1985.

14. J. Steenbrink, Limits of Hodge structures, Invent. Math. 31 (1976), 229-257.

Department of MAthematics, University of Washington, Seattle, WASHINGTON 98195 